\title{
Blinded Status at SDTM Dataset Generation
}

National Cancer Institute

\section{Source}

National Cancer Institute. Blinded Status at SDTM Dataset Generation. NCI Thesaurus.

Code C139272.

The blinding status of the trial at the point in time when the SDTM datasets are generated. 